\title{
Positron Anomaly: Single SNR Explanation without DM or Pulsar Contributions
}

\author{
M.A. Malkov* \\ University of California, San Diego, USA \\ E-mail: mmalkovducsd.edu \\ P.H. Diamond \\ University of California, San Diego, USA
}

\section{R.Z. Sagdeev}

University of Maryland, USA

\begin{abstract}
Recent measurements of $e^{+} / e^{-}$ratio in the $1-500 \mathrm{GeV}$ range were accurate enough to challenge the "standard" model of CR production in SNRs. After a brief decline between $2-8 \mathrm{GeV}$, the ratio rises steadily with a trend towards saturation around $200-400 \mathrm{GeV}$. These observations appear to be in conflict with the diffusive shock acceleration (DSA) mechanism, operating in a single SNR shock, so most explanations appeal to multi-source scenarios. These, however, have a significant number of adjustable parameters.

We argue that $e^{+} / e^{-}$ratio is explicable by a single SNR-DSA if $e^{+}$are produced by shocks which: (i) propagate in clumpy gas media, and (ii) are modified by accelerated CR protons. The protons penetrate into the dense gas clumps upstream to produce positrons and, charge the clumps positively. The induced electric field expels positrons from the clump. These positrons are then accelerated by the modified shock, thus developing a harder spectrum than that of the CR electrons accelerated elsewhere. Mixing these $e^{+}$and $e^{-}$populations explains the increase in the $e^{+} / e^{-}$ratio at $E>8 \mathrm{GeV}$. Its decrease at $E<8 \mathrm{GeV}$ is due to a subshock weakening that results from the shock modification. The subshock weakness also explains why the neutral gas clumps survive its ionizing radiation. Contrary to the positively charged secondary $e^{+}$expelled from the dense gas clumps and accelerated by the DSA mechanism, negatively charged $e^{-}$, in part also $\bar{p}$ along with heavier positive secondaries, such as boron, are locked in the clumps. The weakened subshock engulfs the clumps, so the locked particles evade acceleration. Scenarios for the 100-300 GeV AMS-02 fraction possibly exceeding the model prediction, including possible dark matter and pulsar contributions, are briefly discussed.
\end{abstract}

35th International Cosmic Ray Conference - ICRC2017

10-20 July, 2017

Bexco, Busan, Korea

\footnotetext{
* Speaker.
} 


\section{Overview of Observations and Possible Explanation of the Anomaly}

The latest debates on cosmic ray (CR) spectral anomalies seem to refocus on a perplexing rise of the $e^{+} / e^{-}$fraction in the $10-300 \mathrm{GeV}$ range. The new twist to this anomaly is a possibly related flatness of $\bar{p} / p$ and $e^{+} / p$ ratios. It gained wide interest, especially after the AMS-02 team has published the most accurate elemental and energy spectra in this range [1]]. Anomalies in the CR spectra and composition are thus becoming a general trend in CR observations.

If $e^{+}$and $e^{-}$are accelerated in the same or similar shocks, their spectral differences (by contrast to the secondary positrons not accelerated further in an SNR shock ${ }^{1}$ ) quite plausibly come from charge-sign sensitive acceleration, as their masses are identical. ${ }^{2}$ Most mechanisms for injection, acceleration, and propagation rely on particle interactions with MHD waves, self-driven by the particles or preexisting. At the same time, such interactions do not seem to offer any distinct charge-sign dependence.

However plausible the above argument, the $e^{+} /\left(e^{+}+e^{-}\right)$ratio strongly depends on energy [6, प] (Fig.1], top panel). Moreover, the $e^{+} /\left(e^{+}+e^{-}\right)$ratio possesses one or possibly even two extrema. Strong growth at high energies attracted the most attention, but a distinct minimum at $\approx 8 \mathrm{GeV}$ and descending branch in the range $E<8 \mathrm{GeV}$ may equally be relevant. This nonmonotonic positron fraction is hard to explain with a minimum of assumptions. It appears particularly inconsistent with a single source DSA operation in an SNR, which predicts similar
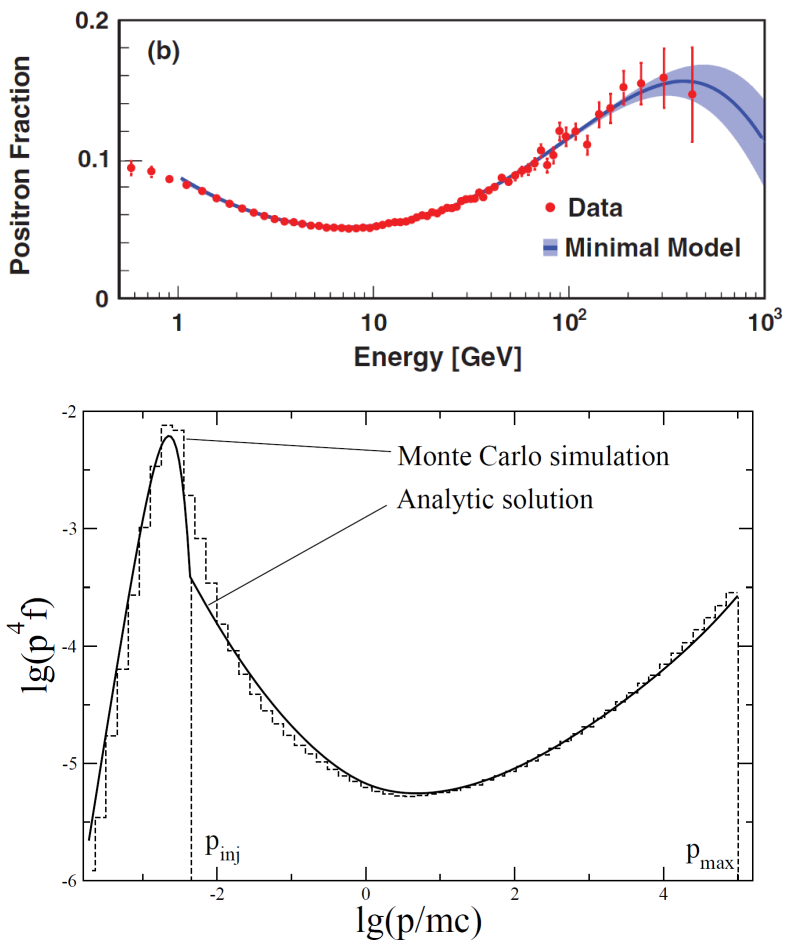

Figure 1: Top Panel: $e^{+} /\left(e^{+}+e^{-}\right)$fraction, as measured by AMS-02 [四].

Bottom Panel: Analytic solution obtained in [] for NLDSA of protons and adopted from [B] (shown together with Monte-Carlo Simulations from [团]).

rigidity spectra for all species accelerated in

the source. The data indicate that this ratio has a trend towards saturation at $200-400 \mathrm{GeV}$, or may even decline beyond this energy range. So, a maximum in this range cannot be currently ruled out which would hint at a possible dark matter (DM) contribution provided that no conventional explanation is given. The goal of this work is to suggest such explanation.

\section{Possible Physics behind the Anomalies}

Accepting charge-sign symmetry of wave-particle interaction, the most likely mechanism be-

\footnotetext{
${ }^{1}$ Note that the injected positrons can be seeded as secondaries, which we assume to be the case and discuss in Sec.[1].

2 cf. p/He spectral anomaly discussed by Hanusch et.al in these Proceedings
} 
hind the $e^{ \pm}$separation during or before the acceleration phase is a macroscopic electric field. Indeed, an accelerator-scale electric field can turn positrons toward it while fending off the electrons, or vice versa. Below, we will suggest a mechanism for electric field generation. It should be noted, however, that the positron excess may also hint at an exciting but still speculative involvement of weakly interacting massive particles (WIMPs). The WIMPS are widely believed to be present in the dark sector of the particle theories beyond the standard model [ 8 , $Q$ ] . Decay or annihilation of such particles has been suggested as a possible explanation for the rise in the $e^{+} / e^{-}$ratio, as well as surprisingly flat (for the secondaries) $\bar{p} / p$ ratio, Fig.】.

\section{Modeling the $e^{+} / e^{-}$spectrum}

Model Requirements and Objectives The current AMS-02 $e^{+} / e^{-}$data, Fig.W, have negligible statistical errors in an extended range between $0.5-100 \mathrm{GeV}$. Moreover, the spectrum in this range is rather complex, thus placing tighter constraints on the models. Therefore, a successful fit of the data in this range lends credence to the model predictions beyond it, including an interesting positron excess (and antiproton, for that matter) where the data are not so exact and even nonexistent (e.g., $E \gtrsim 500 \mathrm{GeV}$ ). An incipient bump around $200-300 \mathrm{GeV}$ was suggested to be possibly related to the dark matter annihilation or decay which can be ruled out if the astrophysical explanation is consistent with the data.

Physics behind the Models Most scenarios for $e^{+} / e^{-}$excess invoke secondary positrons. They are produced by galactic $\mathrm{CR}$ protons in hadronic reactions. The $p p$ - collisions may occur in an ambient gas near an SNR accelerator, or immediately in the SNR shock, thus being incorporated into the DSA. Some of these scenarios face the unmatched antiprotons and other secondaries in the data, as discussed, e.g., in [ए]]. More accurate cross sections of $p p$ collisions obtained in recent years did not shed more light on the physics of $e^{+} / e^{-}$anomaly, particularly the minimum at $8 \mathrm{GeV}$ and the $\mathrm{U}$-shape form of

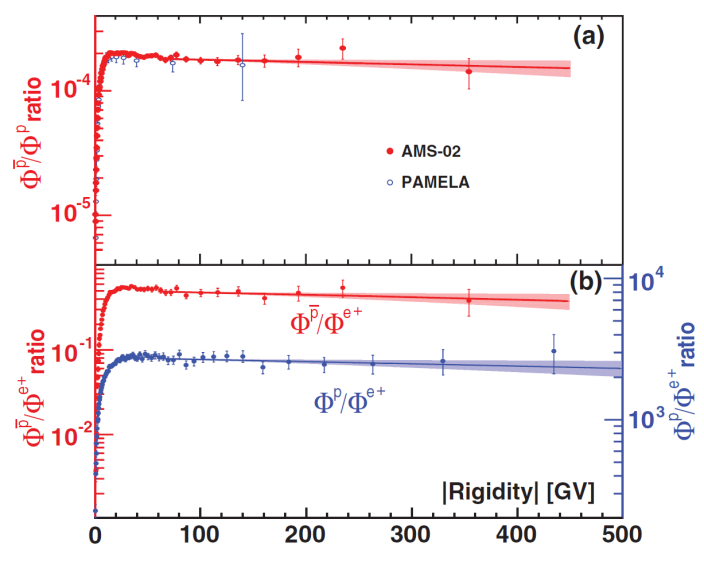

Figure 2: $\bar{p} / p$ and $e^{+} / p$ fractions measured by AMS02 and Pamela. the spectrum, Fig.W. This spectrum complexity hints at richer physics than a mere production of secondary $e^{+}$power-law spectra from the primary CR power-law.

Most of the current models have enough "knobs" to fit the data (see, e.g., [U] for discussion). Some multi-SNR based approaches directly use the AMS-02-measured pair of spectral indices to reproduce the U-shape spectrum in Fig. $\square$ by adjusting the weights of the sources and background contributions. The position of the spectral minimum also needs to be taken directly from the AMS-02 data. Therefore, the minimum is not independently explained by these models, thus giving no credence to their predictions concerning the higher-energy positron excess. Hence, the 
model conclusions about possible room for DM or lack thereof in the positron spectrum remain unsubstantiated.

Of course, before embarking on the quest for DM, one should look for astrophysical interpretations of the $e^{+} / e^{-}$and $\bar{p} / p$ anomalies more carefully. The authors of [W] give an accurate and prosaic interpretation of namely the most precisely measured part of the positron fraction in the range $0.5-100 \mathrm{GeV}$. The explanation is based on the following two aspects of the DSA. First, the mechanism of positron injection is charge-sign asymmetric. They argue that the charge-sign selectivity of injection comes into play when the shock sweeps clumps of dense molecular gas (MC).

The second aspect of the mechanism proposed in [ए]] concerns a nonlinear shock modification by accelerated protons (NLDSA) which is known to make the momentum spectrum of low-energy particles steeper and that of the high-energy particles flatter than the canonical $p^{-4}$ spectrum produced by strong but unmodified shocks. Therefore, there is a point $p=p_{4}$ where the index is exactly four. Assuming most of galactic CR electrons to be accelerated in conventional shocks, thus having $p^{-4}$ source spectra, the ratio of the modified positron- to unmodified electron spectrum will show the required nonmonotonic behavior with a minimum at $p=p_{4}$. Note that it is crucial to work namely with the $e^{+} / e^{-}$ratio, instead of the individual spectra, since the observed spectrum (without possible intervening contributions) is automatically de-propagated back to the source and its surroundings. In a customary $p^{4} f(p)$ normalization, the individual positron spectrum is the same as that of the $e^{+} / e^{-}$ratio which is, in turn, the same as the proton spectrum, provided that both species are accelerated to relativistic energies beyond their injection energies. As the NLDSA is pivotal for the suggested explanation of the positron anomaly, we briefly discuss it below.

\section{Nonlinear Diffusive Shock Acceleration}

The nonlinear proton spectrum is well understood since it is solvable analytically [प2]. Excellent agreement with stationary numerical solutions has been documented, e.g., in [3] , Fig.W. The analytic solution places the proton $p^{4} f(p)$ minimum at $\lesssim 10 \mathrm{GeV} / \mathrm{c}$ which is encouragingly consistent with the position of the observed $e^{+} / e^{-}$minimum at $\approx 8 \mathrm{GeV}$. Moreover, it depends only weakly on the shock Mach number, $M$, proton's maximum momentum, $p_{\max }$, and their injection rate. However, $M \gtrsim 10$ and $p_{\max } / m_{p} c \gtrsim 500$ are the thresholds for a transition to a strongly nonlinear (efficient acceleration) regime, shown in Fig.W, with its signature minimum around 5-10 $\mathrm{GeV}$. A comprehensive understanding of this transition requires an analytic form of the bifurcation diagram, as described, in [[13]. The latter cannot be inferred from stationary numerical solutions. When the solution is not unique, it still converges to one particular branch and does not trace unstable manifolds, required to understand the bifurcation phenomenon. The so-called semi-analytic treatments do not provide the bifurcation diagram (commonly called the S-curve) either, as they do not solve the full problem in analytic form. The significance of the S-curve is that it encompasses three co-existing solutions in a certain range of the proton injection parameter $v_{1}<v_{\text {inj }}<v_{2}$, thus embodying hysteresis in this nonlinear system. It also provides two critical values for injection rates $v_{1,2}$ as functions of the Mach number $M$ and maximum momentum $p_{\max }$. One of the three solutions is unstable. The remaining two are, respectively, an inefficient solution, which corresponds to a test-particle solution at $v_{\text {inj }}<v_{1}$, and the efficient solution. For $v_{i n j}>v_{2}$, this solution is 
unique. Moreover, in the limit $M \gg 1$ and $p_{\max } \gg m_{p} c$ it can be obtained analytically and exactly, which is crucial for obtaining the bifurcation diagram.

The solution co-existence domain between the critical injection rates $v_{1,2}\left(p_{\max }, M\right)$ is typically very narrow, $v_{2}-v_{1} \ll v_{1}$, which additionally prevents stationary numerical solutions from accessing the hysteretic shock behavior. By contrast, time-dependent numerical treatments should easily identify the transition from the inefficient branch at $v_{\text {inj }}<v_{1}$ to efficient one at $v_{\text {inj }}>v_{2}$. Indeed, when the system is evolved from a low $p_{\max } \lesssim m_{p} c$, at first, it sticks with the inefficient solution. With the (slowly) growing $p_{\max }$, the solution passes through the co-existence region, since both $v_{1}$ and $v_{2}>v_{1}$ decrease with $p_{\max }$. As soon as $v_{2}$ becomes $v_{2}<v_{\text {inj }}$, the only remaining system attractor (stationary solution under fixed $p_{\max }$ ) is the efficient solution, to which the numerical time-dependent solution will quickly converge. This type of dynamics was likely seen in timedependent kinetic simulations [14]]. It occurred there in form of a series of sudden jumps to higher shock pre-compressions, manifesting the transitions from inefficient to efficient solutions when the former cease to exist $\left(v_{\mathrm{inj}}>v_{2}\right)$. This pattern of the shock evolution was predicted in [एँ3], including back transitions, though the authors of [14] attributed it to a likely numerical artefact. The incipient back transitions make the time-dependent evolution oscillatory in character, as seen in the simulations. The parameters of these limit-cycle oscillations (related to a Hopf bifurcation) depend on how other control parameters (primarily $v_{\text {inj }}$ ) respond to the changing shock compression during the forward or back transitions. This feedback loop is self-consistently included in the above simulations. Therefore, the limit-cycle type oscillations obtained in [44] are likely to be genuine and worth further study. It will help to understand the NLDSA dynamically, thus shedding new light on many shock-related astrophysical sources where a short-time variability is observed.

\section{Interaction of Accelerated Protons with MCs and Acceleration of Secondaries}

Due to the sub-shock weakening in the NLDSA, MCs survive the ionizing radiation. Moreover, shock-accelerated CR protons illuminate the MC well before the subshock encounter, Fig[3]. These CRs generate positrons and other secondaries in the $\mathrm{MC}$ interior by $p p$ collisions. They also charge the MC positively which creates a chargesign asymmetry for the subsequent particle injection into the DSA via the following simple mechanism [ए]].

Because of a positive electrostatic potential in an MC, built by penetrating shockaccelerated protons, low-energy positively charged particles will be expelled from the MC, while negatively charged particles stay inside. Negatively-charged low-energy sec-

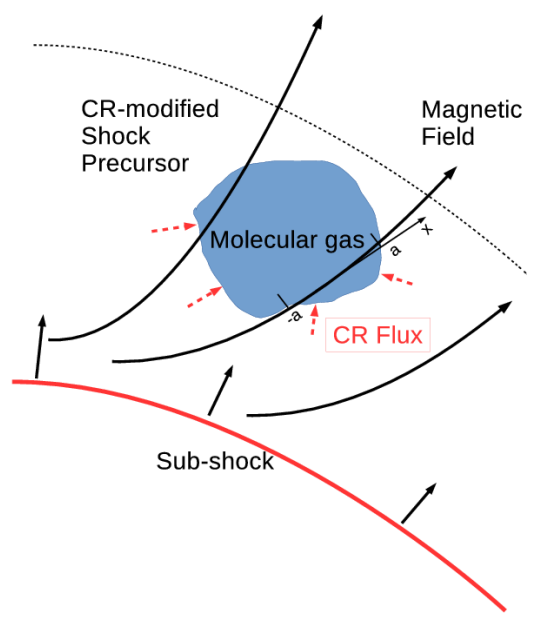

Figure 3: SNR shock propagating into ISM with MC upstream. ondaries produced at the periphery of the MC by relatively low-energy but abundant CR protons will be sucked into the MC. This phe- 
nomenon is particularly consistent with the $\bar{p} / p$ decline towards lower energies, Fig.l. However, for kinematic reasons of their generation and large mass ratio $m_{p} / m_{e}$, antiprotons will be absorbed by the MC much less efficiently than electrons. More importantly for the sequel, the same electric field expels the positively charged positrons from the MCs very efficiently. This is also consistent with an increase of the $e^{+} / e^{-}$ratio, towards lower energies Fig.⿴囗十.

The charging of $\mathrm{MC}$ by accelerated protons is a complex phenomenon [ए]] that we will discuss here only briefly. While fully ionized plasmas are intolerant to external charges and immediately restore charge neutrality, sufficiently large and dense MCs respond to such charges differently. Due to a high rigidity of shock-accelerated CR-protons, their density in the MC interior increases almost simultaneously with their density outside, when a CR-loaded shock approaches the MC. However, by contrast with a strongly ionized exterior, where the plasma resistivity is negligible, the electron-ion collisions inside the MC provide sufficient resistivity to neutralizing electric currents. Therefore, a strong macroscopic electric field will build up in response to the CR penetration, to neutralize the CR charge. This field expels the secondary positrons most efficiently as the lightest positively charged species - although it also shields the MC from low-energy CR protons, thus regulating the production of the secondaries and $\mathrm{MC}$ ionization.

The mechanism outlined above implies that negatively charged primaries and secondaries have much better chances to stay in an MC than positively charged particles. When the subshock eventually reaches the MC, the subshock engulfs it, e.g., [15]]. What was in the $\mathrm{MC}$ interior, is transferred downstream, largely unprocessed by the subshock. Therefore, the negatively charged particles mostly evade acceleration which explains why there is no $\bar{p} / p$ excess similar to that of $e^{+} / e^{-}$at

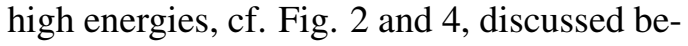
low. Also, antiprotons are strongly depleted towards lower energies, since such $\bar{p}^{\prime} s$ are likely to be trapped by the MC electrostatic potential, as discussed above. Antiprotons born in $p p$ collisions with higher energies (typically a few $\mathrm{GeV}$ ) can be retained in the MC only if they are deep inside. Otherwise, they are accelerated just the way the protons

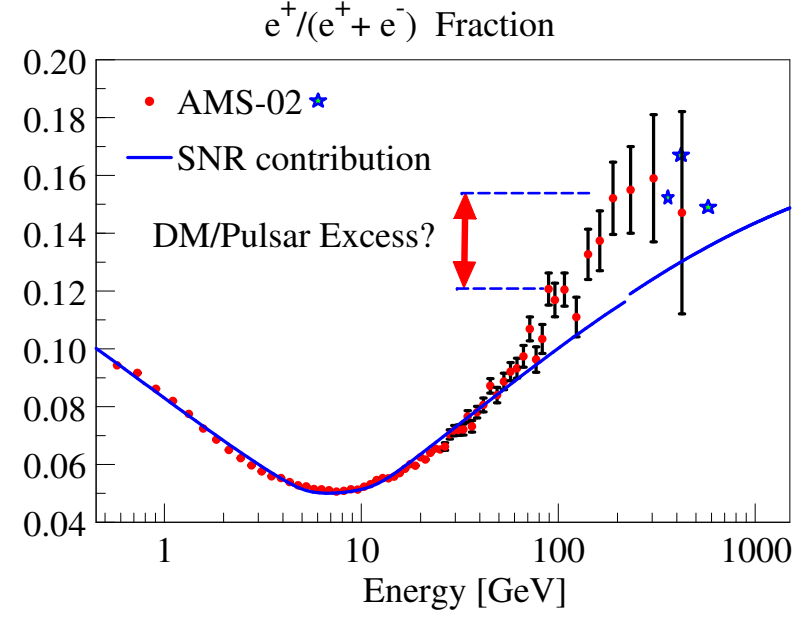

Figure 4: AMS-02 data [四, with the error bars added only where they are significant $(E>30 \mathrm{GeV})$. A few recent points (stars) from $<$ http://www.ams02.org/wpcontent/uploads/2016/12/Final.pdf> are included. Also shown is the positron fraction, obtained by solving the nonlinear acceleration problem. The predicted saturation level $\approx 0.17$. are, which explains the flat $\bar{p} / p$ ratio at higher energies.

An U-curve representing the analytic NLDSA solution described in detail elsewhere [W] is shown in Fig.⿴囗十 It fits well to the AMS-02 data over more than two decades in energy. Other than the normalization of $e^{ \pm}$, and the subshock Mach number, no free parameters, such as weights of different sources, propagation parameters, etc., have been introduced. 


\section{Discussion and Outlook}

The common belief that the CR rigidity spectrum is a power-law with only a few distinct structures (primarily, knee and ankle), the same for all primary elements, is being rapidly abandoned. New data have challenged the idea of acceleration scalability on the entire rigidity range of a given accelerator, such as an SNR. The acceleration in these objects is a multifaceted process.

Multiple sources (SNRs) with adjustable spectra or sources with poorly known spectra (DM, pulsars, superbubbles in uncertain environments) were almost universally proposed to accommodate the new spectral features. By contrast, this paper, and the paper by Hanusch et al. in these Proceedings about the $\mathrm{p} / \mathrm{He}$ anomaly, suggest that a single-SNR CR acceleration successfully accounts for rigidity spectra of different elements with no adjustable model parameters. Moreover, some SNRs are accessible to direct observations across a broad emission spectrum. The CR production in such sources, including the CR interaction with adjacent MCs [ए]], is therefore reasonably well understood. It was demonstrated in this paper that it is possible to fit a high-fidelity part of the data, provided that the following elements of the DSA physics are addressed

- charge-sign dependent lepton injection

- shock and shock-accelerated proton interactions with clumps of dense gas in SNR environments

- backreaction of accelerated protons on the shock structure, particle injection, and acceleration

It should be clear that the single-source mechanisms equally apply to an ensemble of similar SNRs without introducing additional free parameters.

To conclude, we briefly discuss the prospects for resolving the remaining mismatch with the positron fraction in the $200-400 \mathrm{GeV}$ range, Fig. (4. Assuming that the data, notwithstanding error bars in this range, are statistically significant, one may suggest a few explanations of this deviation. The most exciting one is that the limited-energy-range excess over the SNR positron production manifests a DM annihilation or decay. Ironically, the model described in this paper that was incited to account for the data astrophysically facilitates the DM scenario by providing an SNR "background". It significantly eases the requirements for the positron production by DM particles. Not going deeper into DM scenarios, several conventional ways to explain the deviation, may also be suggested [Ш]. Further elaborations on them may or may not close the remaining gap between the SNR positron production and the AMS-02 data. For now, the possibility of some extra DM or pulsar contribution to the positron excess obtained in this paper cannot be ruled out.

\section{Acknoledgements}

This work was supported by the NASA Astrophysics Theory Program under Grant No. NNX14AH36G.

\section{References}

[1] L. Accardo, M. Aguilar, D. Aisa, A. Alvino, G. Ambrosi, K. Andeen et al., High Statistics Measurement of the Positron Fraction in Primary Cosmic Rays of 0.5-500 GeV with the Alpha 
Magnetic Spectrometer on the International Space Station, Physical Review Letters 113 (Sept., 2014) ए210].

[2] M. A. Malkov, Analytic Solution for Nonlinear Shock Acceleration in the Bohm Limit, Astrophys. J. 485 (\#aug\#, 1997) 638-654.

[3] I. V. Moskalenko, T. A. Porter, M. A. Malkov and P. H. Diamond, Hadronic Gamma Rays from Supernova Remnants, ArXiv e-prints 705 (\#may\#, 2007), [0705.3854].

[4] D. C. Ellison, E. G. Berezhko and M. G. Baring, Nonlinear Shock Acceleration and Photon Emission in Supernova Remnants, Astrophys. J. 540 (Sept., 2000) 292-307.

[5] M. Aguilar, L. Ali Cavasonza, B. Alpat, G. Ambrosi, L. Arruda, N. Attig et al., Antiproton Flux, Antiproton-to-Proton Flux Ratio, and Properties of Elementary Particle Fluxes in Primary Cosmic Rays Measured with the Alpha Magnetic Spectrometer on the International Space Station, Physical Review Letters 117 (Aug., 2016) 091103.

[6] O. Adriani, G. C. Barbarino, G. A. Bazilevskaya, R. Bellotti, M. Boezio, E. A. Bogomolov et al., An anomalous positron abundance in cosmic rays with energies 1.5-100Ge V, 458(Apr., 2009) 607-609, 0810.4995 ].

[7] M. Aguilar, G. Alberti, B. Alpat, A. Alvino, G. Ambrosi, K. Andeen et al., First Result from the Alpha Magnetic Spectrometer on the International Space Station: Precision Measurement of the Positron Fraction in Primary Cosmic Rays of 0.5-350 GeV, Physical Review Letters 110(Apr., 2013) 141102.

[8] N. Arkani-Hamed, D. P. Finkbeiner, T. R. Slatyer and N. Weiner, A theory of dark matter, 79(Jan., 2009) 015014, [0.8

[9] V. Berezinsky, V. Dokuchaev and Y. Eroshenko, SUSY dark matter annihilation in the Galactic halo, Dournal of Physics Conference Series 607 (May, 2015) 012015, [1506.03955].

[10] I. V. Moskalenko, A. W. Strong, J. F. Ormes and M. S. Potgieter, Secondary Antiprotons and Propagation of Cosmic Rays in the Galaxy and Heliosphere, Astrophys. J. 565 (Jan., 2002) 280-296, |astro-ph/0106567].

[11] M. Malkov, P. Diamond and R. Sagdeev, Positive charge prevalence in cosmic rays: Room for dark matter in the positron spectrum, Physical Review D 94 (2016) 063006.

[12] M. A. Malkov and L. O. Drury, Nonlinear theory of diffusive acceleration of particles by shock waves, Reports on Progress in Physics 64 (\#apr\#, 2001) 429-481.

[13] M. A. Malkov, Bifurcation, Efficiency, and the Role of Injection in Shock Acceleration with the Bohm Diffusion, Astrophys. J. 491 (\#dec\#, 1997) 584-595.

[14] H. Kang and T. W. Jones, Acceleration of Cosmic Rays at Large Scale Cosmic Shocks in the Universe, Dournal of Korean Astronomical Society 35 (Dec., 2002) 159-174, [astro-ph/0211360].

[15] B. Draine, Physics of the Interstellar and Intergalactic Medium. Princeton Series in Astrophysics. Princeton University Press, 2010.

[16] H. E. S. S. Collaboration, :, H. Abdalla, H. Abdalla, A. Abramowski, F. Aharonian et al., H.E.S.S. observations of RX J1713.7-3946 with improved angular and spectral resolution; evidence for gamma-ray emission extending beyond the X-ray emitting shell, ArXiv e-prints (Sept., 2016), [1609.0867]]. 